\title{
Transient monocular visual impairment as an initial symptom of COVID-19 infection in an individual with multiple sclerosis receiving teriflunomide
}

\author{
Mehmet Fatih Yetkin ${ }^{1} \cdot$ Nur Aleyna Yetkin ${ }^{2} \cdot$ Merve Akcakoyunlu $^{1}$ (D) Meral Mirza $^{1}$ \\ Received: 20 September 2020 / Accepted: 27 January 2021 / Published online: 3 February 2021 \\ (C) Fondazione Società Italiana di Neurologia 2021
}

\begin{abstract}
In December 2019, coronavirus disease 2019 (COVID-19) emerged in Wuhan and rapidly spread throughout China. Since the outbreak of the pandemic, in addition to the well-known COVID-19 symptoms, various neurological symptoms have been also described in patients with COVID-19. Here, we report an unusual presentation of COVID-19 infection in a teriflunomide-treated individual with multiple sclerosis (MS) who did not interrupt teriflunomide treatment during the infection. The course of the infection was mild in this case as in other reported teriflunomide-treated individuals with COVID-19. COVID-19's presentation may be unusual in people with MS (pwMS). It can also be concluded that teriflunomide may be considered a safe diseasemodifying treatment option during the pandemic.
\end{abstract}

Keywords COVID-19 · Multiple sclerosis · Teriflunomide

\section{Introduction}

In December 2019, the outbreak of a novel coronavirus disease (COVID-19) in China spread worldwide and turned into a pandemic which caused great concern [1]. Severe acute respiratory syndrome coronavirus 2 (SARS-CoV-2) is a microorganism that primarily targets the respiratory system. However, due to its neuroinvasive ability, it can also cause symptoms and signs involving the central nervous system $[2$, 3]. Although COVID-19 is most frequently encountered with the symptoms of a fever, cough, and weakness, it may present with symptoms related to neurological involvement such as headaches, consciousness change, ischemic or hemorrhagic infarction, myelitis, myositis, and encephalitis [2, 4].

Data from both clinical and animal studies has shown that coronaviruses (CoVs) can cross the blood-brain barrier and

Merve Akcakoyunlu

merveakcakoyunlu@hotmail.com

1 Department of Neurology, School of Medicine, Erciyes University, 38038 Kayseri, Turkey

2 Department of Pulmonary Medicine, Ministry of Health Kayseri City Hospital, Kayseri, Turkey show neuroinvasive properties [5]. Although the mechanism of invasion into the central nervous system is not fully understood, it is thought that there are four conduction pathways through the olfactory nerve, the passage of lymphocytes and monocytes infected by $\mathrm{CoVs}$, the microvascular endothelium pathway, and the transsynaptic pathway from the peripheral nerves [5].

Multiple sclerosis (MS) is a chronic, persistent inflammatorydemyelinating disease of the central nervous system (CNS) pathologically characterized by areas of inflammation, demyelination, axonal loss, and gliosis [6]. During the pandemic, maintaining a balance between the side effects of any diseasemodifying treatments and the control of the disease activity itself makes the management of MS difficult. In previous studies, it has been shown that pwMS who receive disease-modifying drugs are more likely to develop infections and require hospitalization. The infections have a higher rate of mortality compared to those contracted among the public [7]. During relapsing MS, recently developing neurological symptoms lasting at least $24 \mathrm{~h}$ in the absence of an infection or fever are usually considered to be a new episode of active demyelination [8]. Systemic infections can trigger disease activity and consequently cause relapse or worsen the pre-existing MS symptoms, which is considered to be a pseudo-relapse [7]. Here, we report a teriflunomidetreated individual with MS with an unusual clinical presentation 
of COVID-19 who admitted with monocular visual impairment and consequently had a favorable outcome.

\section{Case report}

A 22-year-old man with no previous medical history presented with a 7-day history of double vision and gait ataxia 2 years ago. The neurological examination was unremarkable except horizontal nystagmus and gait ataxia. Brain magnetic resonance imaging (MRI) demonstrated multiple high signal lesions on the T2 and fluid attenuated inversion recovery (FLAIR) sequences. The cervical MRI was normal except for a slight hyperintensity of the posterior spinal cord at the C3 level on the T2-weighted sequences (Fig. 1). The patient's complaints were almost completely resolved after 5 days of intravenous methylprednisolone treatment. The cerebrospinal fluid (CSF) was positive for oligoclonal bands. He was diagnosed with relapsing remitting multiple sclerosis (RRMS) according to the McDonald criteria and teriflunomide was initiated. There was no evidence of clinical or radiological disease activity for 3 years.
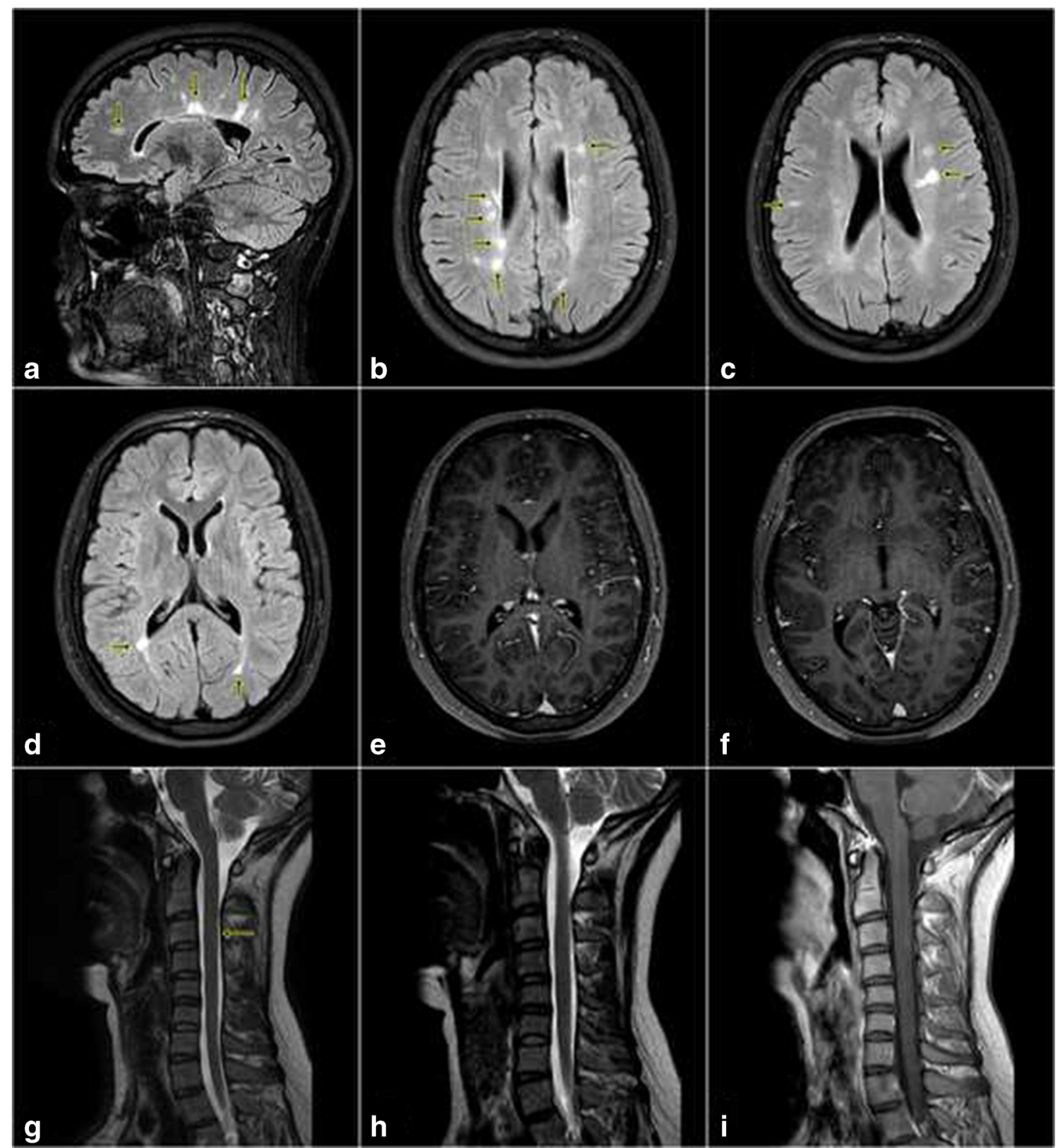

Fig. 1 a, b, c, d Fluid-attenuated inversion recovery sequences reveal hyperintense lesions in the corpus callosum, periventricular, juxtacortical, and subcortical areas. e, f T1-weighted post-contrast sequences show no gadolinium-enhancing lesions. g, h Cervical T2-weighted sequences

show no abnormality except slight hyperintensity of the posterior spinal cord at $\mathrm{C} 3$ level. i Cervical T1-weighted post-contrast sequences show no gadolinium-enhancing lesions 
He was admitted in July 2020 with a visual impairment in his right eye for 5 days during the pandemic. He also started to experience fatigue, headaches, and a loss of smell, and taste 3 days before admission. The vital findings of the patient were as follows: temperature $38.5{ }^{\circ} \mathrm{C}$, heart rate $96 / \mathrm{min}$, oxygen saturation $98 \%$, and blood pressure $125 / 80 \mathrm{mmHg}$.

Following the ophthalmic examination, his visual acuity was evaluated as $8 / 10$ in the right eye and $10 / 10$ in the left eye. Ishihara color plate vision was $10 / 17$ in the right eye and $17 / 17$ in the left eye. Bilateral retinal and optic disc examination was normal. Visual evoked potential (VEP) testing of the right eye showed slightly increased latency but normal amplitude.

The neurological examination revealed slight gait ataxia. The laboratory tests on admission were as follows: C-reactive protein $19 \mathrm{mg} / \mathrm{L}$ (normal, $0-5 \mathrm{mg} / \mathrm{L}$ ), procalcitonin $0.61 \mathrm{ng} /$ $\mathrm{mL}$ (normal, $0-0.5 \mathrm{ng} / \mathrm{mL}$ ), leukocyte $11.9110^{3} / \mu \mathrm{L}$ (normal, $4.8-10.710^{3} / \mu \mathrm{L}$ ), lymphocyte $1.2110^{3} / \mu \mathrm{L}$ (normal, 1.3-2.9), and an erythrocyte sedimentation rate of $24 \mathrm{~mm} / \mathrm{s}$ (normal, 320). A brain MRI was performed and there were no gadolinium-enhancing lesions. There were also no new or enlarging T2 lesions observed compared with the MR images of the previous year. A nasopharyngeal swab was taken for a real-time PCR due to the suspicion of a COVID-19 infection, and the thorax tomography was evaluated as normal. The deterioration in the neurological condition was evaluated as a pseudo-relapse associated with fever therefore intravenous pulse steroid therapy was not administered. Intravenous paracetamol was administered to control the patient's fever. He reported an almost complete improvement in visual the impairment after $12 \mathrm{~h}$. His visual acuity and color vision were found to be within the normal limits on repeated visual examination. Roughly $24 \mathrm{~h}$ later, the nasopharyngeal swab PCR test was found to be positive for COVID-19. Oseltamivir, ciprofloxacin and hydroxychloroquine were initiated and the teriflunomide treatment was not interrupted. His symptoms gradually improved while he was self-quarantined at home, and 25 days after the COVID-19 symptom onset, he was symptom-free. Two follow-up swabs performed $48 \mathrm{~h}$ apart were found to be negative for COVID-19.

\section{Discussion}

The neurological symptoms of COVID-19 infection such as headaches, impaired consciousness, ataxia, acute cerebrovascular disease, seizures, hyposmia, hypogeusia, and neuralgias have also been described in addition to well-defined initial symptoms such as fever, cough, myalgia, and shortness of breath [3]. Wilhelm Uhthoff, a German ophthalmologist, first described the reversible, transient blurring of vision in patients with multiple sclerosis during exercise. Subsequently, it was understood that this loss of vision can occur in other situations such as hot baths, menstruation, and high external temperatures [9]. Although the exact mechanism of the Uhthoff phenomenon (UP) is not understood, it is caused by structural and physiological changes in the demyelinated axons due to a high body temperature [10]. Visual loss as UP is often observed in patients with previous optic neuritis in the affected eye because of the conduction block in the previously demyelinated optic nerve. Interestingly, our patient had never had optic neuritis previously. This can be explained by the silent optic nerve involvement in MS. Davion et al. [11] showed that an optic nerve lesion was present in half of the patients with MS without optic neuritis on 3D double inversion recovery MRI. We thought that the monocular visual impairment in this patient was fever-associated UP which is the result of the systemic inflammatory response rather than a direct central nervous system (CNS) invasion due to COVID-19. Detailed CSF analysis including COVID-19 qualitative real-time reverse-transcriptase-PCR could have been useful to rule out CNS invasion. Unfortunately, we were unable to perform CSF analysis during the acute COVID-19 infection. Also, detailed MR imaging of the optic nerves could have been more useful in understanding the nature of the visual impairment, which is a limitation of our study.

Disease-modifying treatments are widely used to prevent relapses and disability progression in MS. These immunomodulatory agents make pwMS more susceptible to infections compared to the general population $[12,13]$. As a result of the infection, elevated body temperature may temporarily worsen neurological findings [12]. It is considered to be safe to start or continue treatment with glatiramer acetate, teriflunomide, interferon betala- $1 \mathrm{~b}$, dimethyl fumarate, and natalizumab during the COVID-19 pandemic. Fingolimod poses a moderate risk for COVID-19 infection. Alemtuzumab, rituximab, ocrelizumab, and cladribine continue to pose a significant risk [14]. Previous studies have shown that the overactivation of the innate immune response or an inadequate innate and adaptive immune response is associated with poorer outcomes [7]. Teriflunomide selectively and reversibly inhibits dihydro-orotate dehydrogenase, a key mitochondrial enzyme in the de novo pyrimidine synthesis pathway, without either compromising the adaptive immunity or increasing the incidence of serious infections in long-term follow-up [15, 16]. Maghzi et al. [7] concluded that teriflunomide could dampen the unwanted host immune activation and potentially interfere with the viral replication in infected cells by blocking the de novo pyrimidine synthesis and exerting an antiviral effect. It has been reported that the course of infection is often mild in individuals with MS who are using teriflunomide and infected with COVID-19. As recommended, most of the reported pwMS receiving teriflunomide did not interrupt their treatment during the COVID-19 infection [7, 17, 18]. Similarly, in the case we reported, teriflunomide treatment was not interrupted during the COVID-19 infection. It should be also considered that the patient we reported did not have any of 
the risk factors for poor prognosis of COVID-19. Consequently, the course of the infection was mild, and the patient recovered rapidly without any sequelae.

COVID-19 infection may present with unusual neurologic symptoms in pwMS. New neurological symptoms during COVID-19 infection in pwMS should be evaluated carefully especially in the presence of fever. More attention should be paid to avoid unnecessary corticosteroid treatments. Teriflunomide seems to be safe for COVID-19 and it can be recommended to continue teriflunomide treatment during COVID-19 infection.

\section{Declarations}

Ethics approval and consent to participate Ethical approval is not applicable. A written informed consent was obtained.

Conflict of interest There is no conflict of interest.

\section{References}

1. Zhai P, Ding Y, Wu X, Long J, Zhong Y, Li Y (2020) The epidemiology, diagnosis and treatment of COVID-19. Int J Antimicrob Agents 55(5):105955

2. Asadi-Pooya AA, Simani L (2020) Central nervous system manifestations of COVID-19: a systematic review. J Neurol Sci 413: 116832

3. Montalvan V, Lee J, Bueso T, de Toledo J, Rivas K (2020) Neurological manifestations of COVID-19 and other coronavirus infections: a systematic review. Clin Neurol Neurosurg 194:105921

4. Manji H, Carr AS, Brownlee WJ, Lunn MP (2020) Neurology in the time of COVID-19. J Neurol Neurosurg Psychiatry 91(6):568570

5. Sepehrinezhad A, Shahbazi A, Negah SS (2020) COVID-19 virus may have neuroinvasive potential and cause neurological complications: a perspective review. J Neuro-Oncol 26(3):324-329

6. Karussis D (2014) The diagnosis of multiple sclerosis and the various related demyelinating syndromes: a critical review. J Autoimmun 48-49:134-142

7. Maghzi AH et al (2020) COVID-19 in teriflunomide-treated patients with multiple sclerosis. J Neurol:1-7

8. Thompson AJ, Banwell BL, Barkhof F, Carroll WM, Coetzee T, Comi G, Correale J, Fazekas F, Filippi M, Freedman MS, Fujihara K, Galetta SL, Hartung HP, Kappos L, Lublin FD, Marrie RA,
Miller AE, Miller DH, Montalban X, Mowry EM, Sorensen PS, Tintoré M, Traboulsee AL, Trojano M, Uitdehaag BMJ, Vukusic S, Waubant E, Weinshenker BG, Reingold SC, Cohen JA (2018) Diagnosis of multiple sclerosis: 2017 revisions of the McDonald criteria. Lancet Neurol 17(2):162-173

9. Jain A, Rosso M, Santoro JD (2020) Wilhelm Uhthoff and Uhthoff's phenomenon. Mult Scler 26(13):1790-1796

10. Park K, Tanaka K, Tanaka M (2014) Uhthoff's phenomenon in multiple sclerosis and neuromyelitis optica. Eur Neurol 72(3-4): 153-156

11. Davion JB, Lopes R, Drumez É, Labreuche J, Hadhoum N, Lannoy J, Vermersch P, Pruvo JP, Leclerc X, Zéphir H, Outteryck O (2020) Asymptomatic optic nerve lesions: an underestimated cause of silent retinal atrophy in MS. Neurology 94(23):e2468-e2478

12. Boziki MK, Mentis AA, Shumilina M, Makshakov G, Evdoshenko E, Grigoriadis N (2020) COVID-19 Immunopathology and the Central Nervous System: Implication for Multiple Sclerosis and Other Autoimmune Diseases with Associated Demyelination. Brain Sci 10(6):345

13. Kalincik T (2015) Multiple sclerosis relapses: epidemiology, outcomes and management. A systematic review. Neuroepidemiology 44(4):199-214

14. Hamdy SM, Abdel-Naseer M, Shehata HS, Hassan A, Elmazny A, Shalaby NM, Abokrysha NT, Kishk NA, Nada MAF, Ahmed SM, Hegazy MI, Mekkawy D, Mourad HS, Abdelalim A, Berger T (2020) Managing disease-modifying therapies and breakthrough activity in multiple sclerosis patients during the COVID-19 pandemic: toward an optimized approach. Ther Clin Risk Manag 16: 651-662

15. Bar-Or A, Pachner A, Menguy-Vacheron F, Kaplan J, Wiendl H (2014) Teriflunomide and its mechanism of action in multiple sclerosis. Drugs 74(6):659-674

16. Comi G, Miller AE, Benamor M, Truffinet P, Poole EM, Freedman MS (2020) Characterizing lymphocyte counts and infection rates with long-term teriflunomide treatment: pooled analysis of clinical trials. Mult Scler 26(9):1083-1092

17. Mantero V, Baroncini D, Balgera R, Guaschino C, Basilico P, Annovazzi P, Zaffaroni M, Salmaggi A, Cordano C. Mild COVID-19 infection in a group of teriflunomide-treated patients with multiple sclerosis. J Neurol. https://doi.org/10.1007/s00415020-10196-9

18. Möhn N et al (2020) Mild COVID-19 symptoms despite treatment with teriflunomide and hi3gh-dose methylprednisolone due to multiple sclerosis relapse. J Neurol 1-3

Publisher's note Springer Nature remains neutral with regard to jurisdictional claims in published maps and institutional affiliations. 